\title{
EFEITO DO ETHEPHON SOBRE A BROTAÇÃO E VIGOR DOS RAMOS DA VIDEIRA 'NIAGARA ROSADA' (Vitis labrusca L.) $)^{1}$
}

\author{
ANTONIO AUGUSTO FRACARO² \& FERNANDO MENDES PEREIRA ${ }^{3}$
}

\begin{abstract}
RESUMO - A produção da videira 'Niagara Rosada' em regiões tropicais e subtropicais do Brasil tem sido freqüentemente prejudicada, principalmente devido à dificuldade de emissão e desenvolvimento das brotações após a poda de produção, realizada nos meses de ocorrência de temperaturas mais baixas, o que tem causado redução nas produções e desestímulo aos viticultores. Para solucionar esse problema, foram conduzidos três experimentos, em pomares comerciais localizados na região Noroeste do Estado de São Paulo, com o objetivo de estudar o efeito do Ethephon, aplicado antes da poda de produção, na emissão e desenvolvimento das novas brotações e na duração do período da poda até a floração. Foram testadas quatro doses de ethephon (0 mg.L. $\left.\mathrm{L}^{-1} ; 720 \mathrm{mg} . \mathrm{L}^{-1} ; 1.440 \mathrm{mg} . \mathrm{L}^{-1} ; 2.160 \mathrm{mg} . \mathrm{L}^{-1}\right)$ aplicadas via foliar antes da poda de produção, nos meses de junho e julho de 2002. Observou-se que a aplicação de Ethephon proporcionou maior número de gemas brotadas, maior comprimento e diâmetro do ramo e não alterou o período da poda à floração. Especialmente quando da ocorrência de condições climáticas desfavoráveis e quando as plantas apresentaram satisfatório grau de enfolhamento, a aplicação de ethephon, na dose de $2.160 \mathrm{mg} . \mathrm{L}^{-1}$, foi a mais efetiva.
\end{abstract}

Termos de indexação: brotação, reguladores vegetais, uva, videira

\section{EFFECT OF ETHEPHON ON SPROUT AND VIGOR OF BRANCH OF 'NIAGARA ROSADA' (Vitis labrusca L.)}

\begin{abstract}
The production of 'Niagara Rosada ' in tropical and subtropical regions of Brazil has been frequentmently reduced, mainly due to difficulty of emission and development of sprout after the yield prune carried out in the months of occurrence of lower temperatures, what caused reduction in yields and discourage of farmers. Three experiments was carried out to solve this problem, in commercial areas located in northwest region of São Paulo State, with the objective to verify the effect of Ethephon, applied before the yield prune, in emission and development of sprout and in duration of the period of pruning until the flowering. Four levels of Ethephon $\left(0 \mathrm{mg} . \mathrm{L}^{-1} ; 720 \mathrm{mg} . \mathrm{L}^{-1} ; 1,440 \mathrm{mg} . \mathrm{L}^{-1} ; 2,160 \mathrm{mg} . \mathrm{L}^{-1}\right)$ were applied by leaf before the yield prune, in the months of June and July of 2002. It was observed, that the application of Ethephon promoted greater sprouted bud number. Greater growth and diameter of branch and did not modify the duration of the period of pruning until the flowering. Speciallity when the climatic conditions, were unfavorable and when the plants present satisfactory level of leaf, it recommends application of Ethephon in the dose of $2,160 \mathrm{mg} . \mathrm{L}^{-1}$, this revealed be more effective.
\end{abstract}

Index Terms - sprout, growth regulator, grape,

\section{INTRODUÇÃO}

A viticultura brasileira abrange uma área de 63.816ha. No Estado de São Paulo, 9.082ha são utilizados na produção de uvas, sendo 5.270,2ha de 'Niagara Rosada', dos quais a região Noroeste possui 108,5ha (Corrêa et al., 2000; Protas et al., 2002).

A 'Niagara Rosada' representa cerca de $28 \%$ do volume de uvas comercializadas na CEAGESP-SP (Cato et al., 1999).

A região Noroeste de São Paulo cultiva, principalmente, uvas finas de mesa (Vitis vinifera L.), como 'Itália', 'Rubi', 'Benitaka' e 'Brasil', que apresentam um elevado custo de produção, em virtude da necessidade intensa de mão-de-obra e de tratamentos fitossanitários. Tal fato tem levado muitos produtores a optarem pelo plantio de outras variedades, dentre as quais a 'Niagara Rosada'.

Esta variedade tem se apresentado como ótima alternativa para a região, devido ao menor custo com mão-de-obra (por não necessitar de raleio de bagas), menor suscetibilidade às doenças fúngicas, boa aceitação no mercado interno e aos bons rendimentos obtidos em condições irrigadas (Conceição et al., 1999).

Normalmente, a época de colheita ocorre na entressafra das regiões produtoras tradicionais, quando os preços são mais elevados. A colheita, quando realizada neste período, correspondendo aos meses de setembro-outubro, tem alcançado preços acima de $\mathrm{R} \$ 1,80 \mathrm{~kg}$ ${ }^{1}$, sendo considerado muito vantajoso.

Entretanto, essa produção tem sido problemática na região, principalmente devido à dificuldade de emissão e desenvolvimento das brotações após as podas, sobretudo quando estas são realizadas nos meses de ocorrência de temperaturas mais baixas (maio a julho), causando conseqüentemente baixas produções.
$\mathrm{Na}$ tentativa de solucionar esses problemas, podem-se utilizar reguladores vegetais, em especial o ethephon. Porém, o modo de ação é dependente do local da síntese ou tecido aplicado, do tempo de síntese ou da aplicação, do nível de ação do composto, bem como da sua interação e a inter-relação funcional de diferentes hormônios e reguladores de crescimento (Korban, 1998).

A síntese de etileno é influenciada por fatores como temperatura, teor carbônico, oxigênio e está correlacionado à presença de outros hormônios e reguladores vegetais (Schiaparelli et al., 1995).

A biossíntese inicia com o aminoácido metionina, que reage com ATP para formar um composto conhecido por Sadenosilmetionina (SAM), sendo quebrado em dois compostos diferentes, um dos quais é o chamado ACC (ácido 1aminociclopropano-1-carboxílico), onde enzimas no tonoplasto convertem o ACC em etileno (Raven et al., 2001).

Na região Noroeste do Estado de São Paulo, Fracaro (2000) utilizou o ethephon em videira 'Rubi' e obteve excelentes resultados para aumentar a intensidade de brotação e o vigor da planta, com uma aplicação, 20 dias antes da poda na dosagem de 5 L.ha $^{-1}$ (1.200 mg. $\left.\mathrm{L}^{-1}\right)$.

Embasado nesses resultados e na tentativa de sanar os problemas de brotação da "Niagara Rosada", foram iniciadas as pesquisas com a utilização do ethephon nessa cultivar, de grande importância para a expansão da cultura, não somente na região, mas também nas demais regiões tropicais e subtropicais.

O objetivo da presente pesquisa foi avaliar o efeito de diferentes doses de ethephon, aplicado via foliar antes da poda de produção, na emissão e desenvolvimento das brotações e na duração do período da poda até a floração da videira 'Niagara Rosada'.

\footnotetext{
${ }^{1}$ (Trabalho 041/2004). Recebido: 16/04/2004. Aceito para publicação: 17/12/2004. Parte da tese de doutorado do primeiro autor a FCAV/UNESP. Trabalho financiado pela FAPESP.

2 Doutorando em Agronomia, Departamento de Produção Vegetal, Universidade Estadual Paulista-UNESP, Faculdade de Ciências Agrárias e Veterinárias- FCAV, Câmpus de Jaboticabal, Rodovia de Acesso Prof. Paulo Donato Castellane, s/n, CEP14884-900, Jaboticabal-SP. Bolsista CNPq. Fone 173621 6884. E-mail: fracaro.fracaro@itelefonica.com.br.

${ }^{3}$ Dr., Prof. Titular Voluntário, Departamento de Produção Vegetal, FCAV/UNESP, Câmpus de Jaboticabal, Jaboticabal-SP. E-mail: nlynn@ fcav.unesp.br.
} 
TABELA 1. Dados meteorológicos diários da região Noroeste do Estado de São Paulo, dos meses de junho e julho de 2002.

\begin{tabular}{|c|c|c|c|c|c|c|c|c|c|c|c|}
\hline \multicolumn{6}{|c|}{ Junho } & \multicolumn{6}{|c|}{ Julho } \\
\hline \multicolumn{3}{|c|}{ Temp. $\left({ }^{\circ} \mathrm{C}\right)$} & \multicolumn{3}{|c|}{ Temp. $\left({ }^{\circ} \mathrm{C}\right)$} & \multicolumn{3}{|c|}{ Temp. $\left({ }^{\circ} \mathrm{C}\right)$} & \multicolumn{3}{|c|}{ Temp. $\left({ }^{\circ} \mathrm{C}\right)$} \\
\hline Dia & Máx & Mín & Dia & Máx & Mín & Dia & Máx & Mín & Dia & Máx & Mín \\
\hline 01 & 30,6 & 17,4 & 16 & 30,4 & 17,0 & 01 & 31,0 & 16,0 & 16 & 28,8 & 15,2 \\
\hline 02 & 30,2 & 16,0 & 17 & 30,0 & 16,0 & 02 & 30,0 & 18,0 & 17 & 32,4 & 15,8 \\
\hline 03 & 30,2 & 17,0 & 18 & 30,8 & 15,8 & 03 & 29,8 & 17,0 & 18 & 31,0 & 16,2 \\
\hline 04 & 30,0 & 16,8 & 19 & 31,0 & 16,0 & 04 & 31,2 & 16,0 & 19 & 32,0 & 18,0 \\
\hline 05 & 30,4 & 15,0 & 20 & 30,4 & 17,4 & 05 & 32,0 & 19,2 & 20 & 31,4 & 16,2 \\
\hline 06 & 29,8 & 17,0 & 21 & 29,4 & 15,6 & 06 & 28,0 & 18,2 & 21 & 23,8 & 15,0 \\
\hline 07 & 31,0 & 17,4 & 22 & 28,0 & 16,0 & 07 & 22,0 & 18,2 & 22 & 19,2 & 13,8 \\
\hline 08 & 31,8 & 19,0 & 23 & 29,0 & 16,4 & 08 & 21,0 & 18,2 & 23 & 28,6 & 17,0 \\
\hline 09 & 31,2 & 18,0 & 24 & 29,8 & 16,8 & 09 & 21,0 & 10,0 & 24 & 31,2 & 14,6 \\
\hline 10 & 32,2 & 18,4 & 25 & 29,6 & 17,0 & 10 & 25,2 & 10,2 & 25 & 32,4 & 17,0 \\
\hline 11 & 32,6 & 16,9 & 26 & 28,8 & 17,0 & 11 & 22,8 & 16,2 & 26 & 31,2 & 15,8 \\
\hline 12 & 31,6 & 15,2 & 27 & 31,0 & 17,4 & 12 & 28,0 & 18,2 & 27 & 32,2 & 15,4 \\
\hline 13 & 31,2 & 15,0 & 28 & 31,6 & 17,8 & 13 & 22,0 & 17,6 & 28 & 24,0 & 8,0 \\
\hline 14 & 31,8 & 18,0 & 29 & 31,0 & 18,2 & 14 & 21,0 & 18,4 & 29 & 28,0 & 15,2 \\
\hline 15 & 31,0 & 16,0 & 30 & 29,0 & 16,8 & 15 & 25,0 & 21,4 & 30 & 32,4 & 18,0 \\
\hline & & & 31 & & & & & & 31 & 32,8 & 17,0 \\
\hline
\end{tabular}

Fonte: CIIAGRO (2003)

\section{MATERIALEMÉTODOS}

Foram realizados três experimentos em vinhedos comerciais de 'Niagara Rosada', implantados sobre porta-enxerto IAC 572-Jales, no sistema de latada, com irrigação por microaspersão e espaçamento $2,5 \mathrm{x}$ 2,0m, na região de Jales, Noroeste do Estado de São Paulo (latitude $20^{\circ} 16^{\prime} \mathrm{S}$, longitude $50^{\circ} 33^{\prime} \mathrm{W}$ ) e altitude média de $483 \mathrm{~m}$ (Terra et al., 1998).

Segundo o sistema de classificação de Köeppen, o clima da região é subtropical úmido, CWa, com inverno seco e ameno, e verão quente e chuvoso (Boliani, 1994).

O delineamento experimental utilizado foi o de blocos ao acaso, com quatro doses de ethephon e cinco repetições de uma planta por parcela.

Os tratamentos utilizados foram:1-Testemunha (sem aplicação de ethephon); 2- ethephon $720 \mathrm{mg} . \mathrm{L}^{-1} ; 3$ - ethephon $1.440 \mathrm{mg} . \mathrm{L}^{-1} ; 4$ ethephon 2.160 mg.L. ${ }^{-1}$, sendo 0 L.ha $^{-1} ; 3$ L.ha $^{-1} ; 6$ L.ha $^{-1}$ e 9 L.ha ${ }^{-1}$ do produto comercial, respectivamente, para cada tratamento.

O produto, diluído em água, foi aplicado sobre as plantas com enfolhamento superior a $70 \%$ através de um pulverizador costal até o ponto de escorrimento (0,5 litro por planta).

As avaliações foram: número de gemas brotadas, sendo avaliada a quantidade de brotos emitidos nas últimas 5 gemas apicais dos ramos podados; comprimento das brotações, medido semanalmente até o período da floração; diâmetro das brotações, medido a $10 \mathrm{~cm}$ da base no período de floração; e a duração em dias do período da poda até a plena floração.

Os tratos culturais, como adubação, irrigação, quebra de dormência com cianamida hidrogenada, controle fitossanitário, podas e outros foram os convencionais adotados na região. Na Tabela 1, são apresentados os dados meteorológicos diários da região Noroeste do Estado de São Paulo, fornecidos pelo CIIAGRO (2003)

\section{RESULTADOS E DISCUSSÃO}

Na Figura 1, observa-se nitidamente a grande diferença de brotação entre os tratamentos; aumentando a dose de ethephon, verificou-se um grande aumento no número de gemas brotadas.

Nos tratamentos sem aplicação de ethephon e com ocorrência de baixas temperaturas no período da brotação, observaram-se resultados inferiores, principalmente no experimento 2 . Neste experimento, ocorreu temperatura mais baixa durante o período de brotação, prejudicial à emissão da brotação e desenvolvimento do ramo. Independentemente das condições climáticas, um maior número de gemas brotadas foi obtido com a utilização do regulador de crescimento, principalmente na dose de $2.160 \mathrm{mg} \cdot \mathrm{L}^{-1}$.

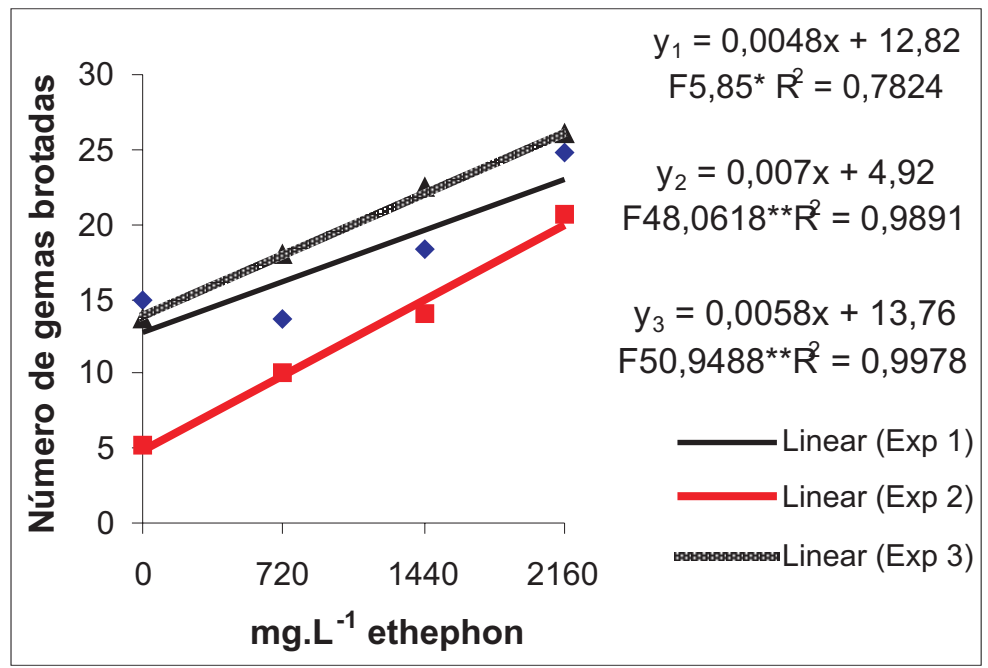

FIGURA 1 - Efeito da aplicação de ethephon antes da poda de produção sobre o número de gemas brotadas da videira 'Niagara Rosada', cultivadas na região Noroeste do Estado de São Paulo, Jales-SP, 2002.

Trabalhando com uva 'Rubi', Fracaro \& Boliani (2001) observaram o aumento da brotação com o aumento da concentração de ethephon, resultados estes semelhantes aos obtidos neste trabalho. Provavelmente, estes resultados estão relacionados ao grande número de folhas presentes no momento da aplicação. Fracaro et al. (2004), trabalhando com a aplicação de ethephon em plantas pouco enfolhadas, não obtiveram resultados estatísticos significativos, porém existe clara tendência de aumento do número de gemas brotadas. De acordo com Ben-Tal et al. (1993), a maior quantidade aplicada de reguladores de crescimento não é absorvida por outro órgão da planta, mas sim pelo tecido-alvo.

Fracaro (2000) salienta a importância de usar o regulador de crescimento, em plantas em bom estado fitossanitário e nutricional, para suportar as modificações fisiológicas ocorridas nas plantas. Por isso, é imprescindível a presença de folhas para haver maior absorção do produto, proporcionando melhores resultados.

A desfolha causada pelo uso do ethephon é uma prática que facilita a poda e provavelmente a translocação de assimilados das folhas para os órgãos de reservas (Fracaro, 2000). 

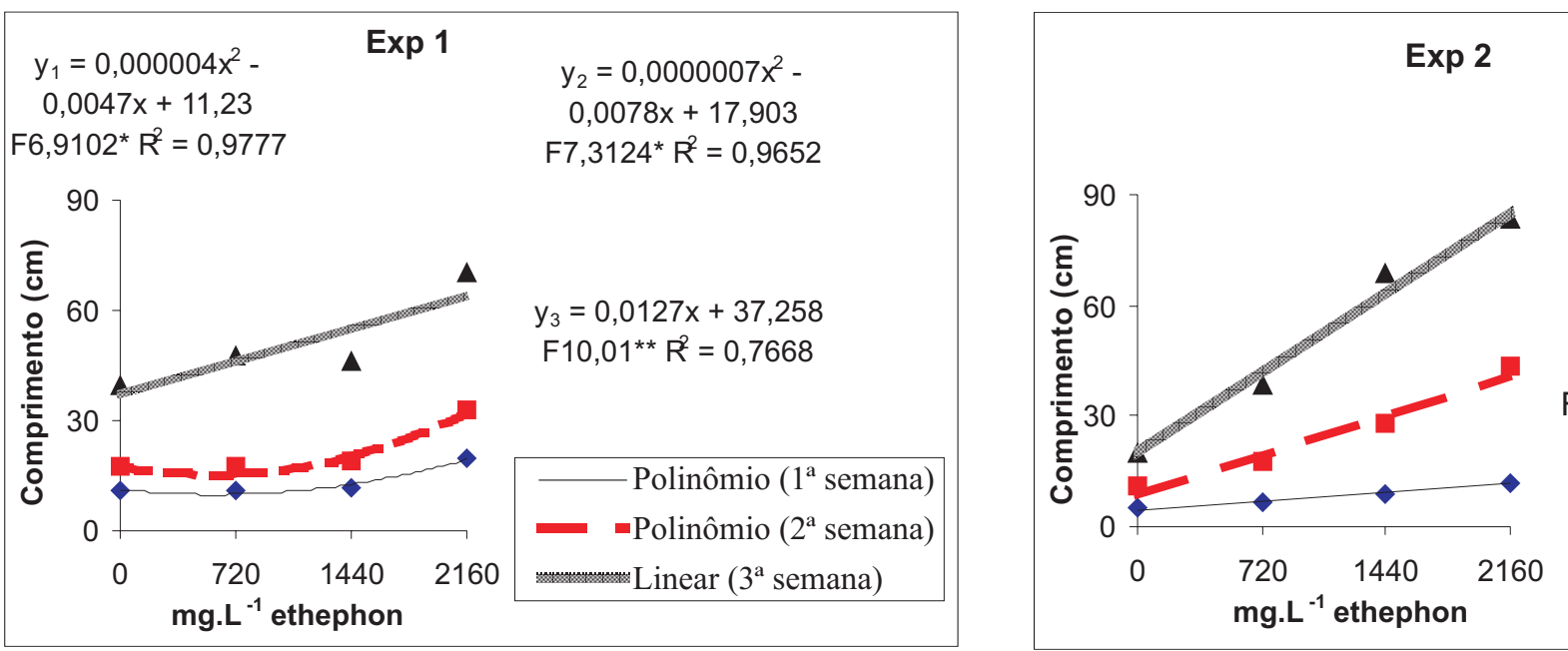

$$
\begin{gathered}
y_{1}=0,0031 x+4,779 \\
F 54,0757^{* *} R^{2}=0,9349
\end{gathered}
$$

$\mathrm{y}_{2}=0,015 \mathrm{x}+8,872$

$F 131,4138^{* *} R^{2}=0,9687$

$\mathrm{y}_{3}=0,0305 \mathrm{x}+19,459$

$\mathrm{F} 218,2306^{* *} \mathrm{R}^{2}=0,9845$

FIGURA 2 - Efeito da aplicação de ethephon antes da poda de produção sobre o crescimento dos ramos da videira 'Niagara Rosada', avaliados semanalmente, cultivada na região Noroeste do Estado de São Paulo, Jales-SP, 2002.

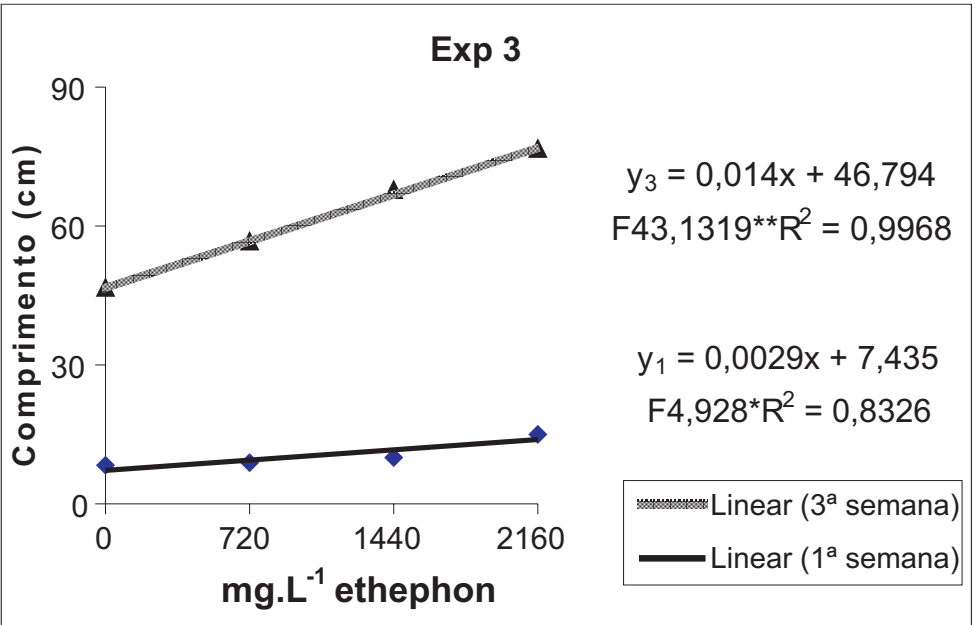

FIGURA 3 - Efeito da aplicação de ethephon antes da poda de produção sobre o comprimento dos ramos da videira 'Niagara Rosada', avaliados semanalmente, cultivada na região Noroeste do Estado de São Paulo, Jales-SP, 2002.

Nas Figuras 2 e 3, pode ser observado o desenvolvimento do comprimento dos ramos, medidos semanalmente no período de brotaçãofloração.

Analisando conjuntamente os experimentos, verifica-se que a testemunha foi inferior quando comparada aos tratamentos com ethephon.

O ethephon, na maior dose, proporcionou, na terceira semana, um desenvolvimento dos ramos com valores próximos para todos os experimentos, independentemente das condições climáticas, ou seja, o comprimento dos ramos tendeu a desenvolver-se muito vigorosamente, obtendo-se, porém, valores finais semelhantes.

No experimento 2 (ocorrência de temperaturas mais baixas no período de brotação), foi nítido o menor crescimento dos ramos na testemunha. Portanto, pode-se concluir que o uso de ethephon promoveu melhor brotação e intenso desenvolvimento do ramo, proporcionando à uva 'Niagara Rosada' grande aumento em seu vigor.

Nos três experimentos, observados na Figura 4, percebe-se o efeito da aplicação de ethephon no diâmetro dos ramos da videira, os quais apresentaram tendência de aumento com o aumento da dose de ethephon. Nos experimentos 2 e 3, foram observadas diferenças estatísticas significativas para as maiores doses entre os tratamentos.

Na Tabela 2, verifica-se que não houve diferença estatística significativa na época de florescimento. Portanto, o uso de ethephon promoveu aumento de vigor, mas não alterou o ciclo da planta. Resultados semelhantes foram obtidos por Fracaro (2000) em uva 'Rubi' e por Fracaro et al. (2004) trabalhando com uva 'Niagara Rosada' com pouco enfolhamento.

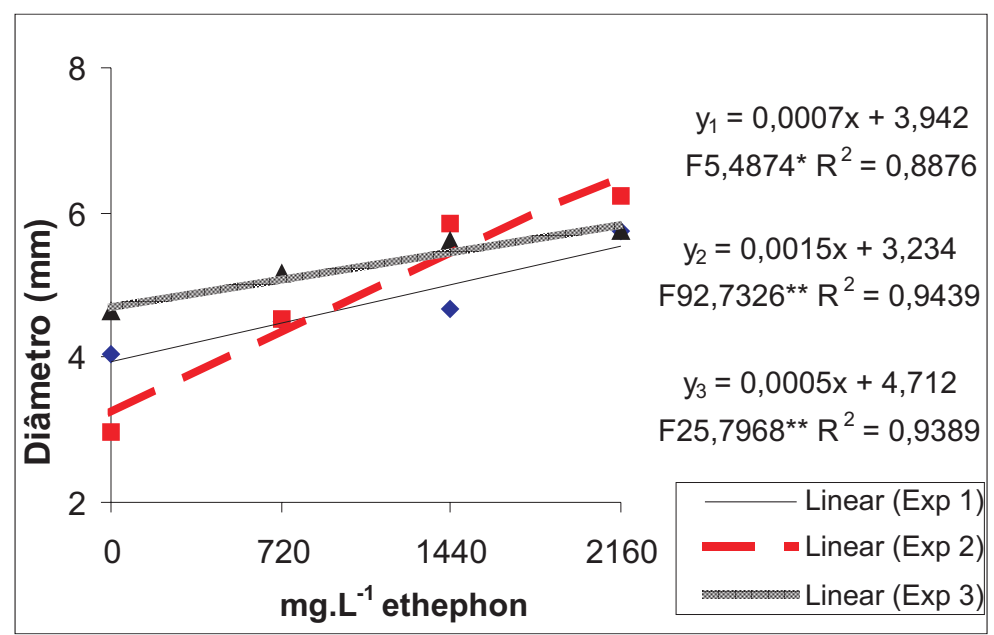

FIGURA 4 - Efeito da aplicação de ethephon antes da poda de produção sobre o diâmetro dos ramos da videira 'Niagara Rosada', cultivada na região Noroeste do Estado de São Paulo, Jales-SP, 2002.

TABELA 2 - Duração em dias do período da poda à floração da videira 'Niagara Rosada', cultivada na região Noroeste do Estado de São Paulo, Jales-SP, 2002.

\begin{tabular}{cccc}
\hline $\begin{array}{c}\text { Tratamentos } \\
\text { mg.. }\end{array}$ & \multicolumn{3}{c}{ Período da Poda à Florada (dias) } \\
\cline { 2 - 4 } & Experimento 1 & Experimento 2 & Experimento 3 \\
\hline Testemunha & 36 & 38 & 34 \\
720 & 36 & 38 & 34 \\
1.440 & 36 & 38 & 34 \\
2.160 & 36 & 38 & 34 \\
\hline
\end{tabular}

É importante salientar que da aplicação do ethephon até o início da emissão da brotação decorre um período de cerca de 30 dias. Isso praticamente inviabiliza qualquer previsão meteorológica sobre a ocorrência de baixas temperaturas que possam prejudicar o desenvolvimento das brotações, o que reforça ainda mais a necessidade de viabilização do uso do ethephon, cuja aplicação causou aumento na emissão e no desenvolvimento das brotações da videira, promovendo aumento do vigor e, conseqüentemente, aumento da produção.

\section{CONCLUSÕES}

Em vinhedos com índice satisfatório de enfolhamento e com ou sem ocorrência de temperaturas amenas na época de brotação, a aplicação de ethephon em cv. Niagara Rosada, antes da poda, permitiu as seguintes conclusões:

1. melhores brotações e excelente desenvolvimento dos ramos; 
2. a dose $2.160 \mathrm{mg} . \mathrm{L}^{-1}$ promoveu o maior número de gemas brotadas, maior crescimento e diâmetro do ramo, especialmente quando da ocorrência de condições climáticas desfavoráveis, abaixo de $10^{\circ} \mathrm{C}$;

3. não houve alteração do período da poda ao florescimento.

\section{REFERÊNCIAS}

BEN-TAL, Y.; WODNER, M. Absorption of plant growth regulators by fruit trees. Acta-Horticulturae, Wageningen, n.329, p 62 - 69, 1993.

BOLIANI, A.C. Avaliação fenológica de videiras Vitis vinifera $\mathbf{L}$. cv. Itália e cv. Rubi, na região Noroeste de São Paulo. 1994. 188f. Tese (Doutorado em Produção Vegetal) - Faculdade de Ciências Agrárias e Veterinárias, Universidade Estadual Paulista, Jaboticabal, 1994.

CATO, C.C.; TERRA, M.M.; PIRES, E.J.P.; BOTELHO, R.V.; STIVARI, A. Efeito do anelamento e doses de ácido giberélico na frutificação da uva 'Niagara Rosada' na região Oeste do Estado de São Paulo. In: CONGRESSO BRASILEIRO DE VITICULTURA E ENOLOGIA, 9. 1999, Bento Gonçalves. Anais... Bento Gonçalves: EMBRAPA, 1999, p.142.

CENTROINTEGRADODE INFORMAÇÃOAGROMETEOROLÓGICA (CIIAGRO), Instituto Agronômico de Campinas. Dados Diários por Local. Disponível em <http://ciiagro.iac.br/dados/Jales>. Acesso em: 27-07-2003.

CONCEIÇÃO, M.A.F.; MANDARINI NETO, J.; MAIA, J.D.G. Evapotranspiração da videira 'Niagara Rosada' em Jales-SP In: CONGRESSO BRASILEIRO DE VITICULTURAE ENOLOGIA, 9., 1999. Bento Gonçalves. Anais... Bento Gonçalves: EMBRAPA, 1999. p.157

CORRÊA, L. de S.; BOLIANI, A.C. O cultivo de uvas de mesa no Brasil e no mundo e sua importância econômica In: SIMPÓSIO BRASILEIRO SOBRE UVAS DEMESA, 2000, Ilha Solteira. Anais...p. $1-34$.
FRACARO, A.A. Efeito de doses crescentes de ethephon em videira 'Rubi'(Vitis vinifera L.), cultivada na região Noroeste do Estado de São Paulo. 2000. 88f. Dissertação (Mestrado em Sistema de Produção) - Faculdade de Engenharia de Ilha Solteira, Universidade Estadual Paulista, Ilha Solteira, 2000.

FRACARO, A.A.; PEREIRA, F.M.; NACHTIGAL, J.C. Uso de ethephon antes da poda de produção em videira 'Niagara Rosada'(Vitis labrusca L.). Revista Brasileira de Fruticultura, Jaboticabal-SP, v.26, n.1, p.97-100, 2004.

FRACARO, A.A.; BOLIANI, A.C. Efeito do ethephon em videira 'Rubi' (Vitis vinifera L.), cultivada na região Noroeste do estado de São Paulo. Revista Brasileira de Fruticultura, Jaboticabal-SP, v.23, n.3, p.510-512, 2001

KORBAN, S.S. Influence of growth regulators on fruit plant growth and development. In: CONGRESSO BRASILEIRO DEFRUTICULTURA, 15., 1998, Poços de Caldas. Anais... Lavras:SBF, 1998. p 56-81.

PROTAS, J.F.S.; CAMARGO, U.A.; MELO, L.M.R. A viticultura brasileira: realidade e perspectivas In: SIMPÓSIO MINEIRO DE VITICULTURA EENOLOGIA, 1, 2002, Caldas. Anais... Caldas: EPAMIG, 2002.p. 1732

RAVEN, P.H.; EVERT, R.F.; EICHHORN, S.E. Biologia vegetal. 6. ed. Rio de Janeiro: Ed. Guanabara Koogan, 2001. 872p.

SCHIAPARELLI, A.; SCHREIBER, G.; BOURLOT, G. Fitoregolatori in Agricoltura: storia, prodotti, impieghi, normative, ricerca e sviluppo. Bolonha:Edagricole, 1995.319p.

TERRA, M.M.; PIRES, E.J.P.; NOGUEIRA, N.A.M. Tecnologia para produção de uva 'Itália' na região Noroeste do Estado de São Paulo. Campinas:CATI, 1998.81p. (Documento técnico, 97). 